\title{
Definition of Shifts of Optical Transitions Frequencies due to Pulse Perturbation Action by the Photon Echo Signal Form
}

\author{
V.N. Lisin ${ }^{1 a}$, A.M. Shegeda ${ }^{1}$, and V.V. Samartsev ${ }^{1,2}$ \\ ${ }^{1}$ Zavoisky Physical-Technical Institute, 420029, Sibirsky Trakt 10/7, Kazan \\ ${ }^{2}$ Kazan (Volga region) Federal University, Cremlin street, 1,Kazan, 420000, Russia
}

\begin{abstract}
A relative phase shift between the different groups of excited dipoles, which appears as result of its frequency splitting due to action of a pulse of electric or magnetic fields, depends on a time, if the pulse overlaps in time with echo-pulse. As a consequence, the echo waveform is changed. The echo time form is modulated. The inverse modulation period well enough approximates Zeeman and pseudo-Stark splitting in the cases of magnetic and, therefore, electrical fields. Thus the g-factors of ground ${ }^{4} \mathrm{I}_{15 / 2}$ and excited ${ }^{4} \mathrm{~F}_{9 / 2}$ optical states of $\mathrm{Er}^{3+}$ ion in $\mathrm{LuLiF}_{4}$ and $\mathrm{YLiF}_{4}$ have been measured and pseudo-Stark shift of R1 line in ruby has been determined.
\end{abstract}

Keywords: photon echo, pulse frequency splitting, phase shift, echo waveform modulation, pseudoStark shift, R1 line, ruby.

In this report we present our new results of pseudo-Stark splitting measurement by method of modulation of photon echo waveform. The results of our works [1-4] concerning the measurements of paramagnetic ion's g-factor by this method with applying of the magnetic pulse are presented for illustration of the possibilities of the method.

Echo intensity oscillates versus the phase [1-4]:

$$
I(t)=I_{0}(t)(1+\cos \phi(t)) / 2, \phi(t)=2 \pi 2 \frac{\partial v}{\partial E} \int_{t_{0}}^{t} E\left(t^{\prime}\right) d t^{\prime}
$$

where the relative phase shift $\varphi(t)$ between the two groups of excited dipoles in the case of magnetic pulse depends on Stark coefficient $\partial v / \partial E$, the area of the electric-field pulse in moment $t\left(t_{0}\right.$ is the time of the beginning of the action of an electric pulse). A magnitude of electric field $E(t)$ is approximated by voltage $V$ divided by sample thickness $d$. Here $I_{0}(t)$ is the intensity of an echo when an electric pulse is off. $E(t)$ must be exchanged by $H(t)$ in (1) if magnetic pulse is applied.

The next curves are shown in Fig.1a: photon-echo intensity $I_{0}(t)$ for excitation of $\mathrm{R}_{1}$ line in ruby, voltage pulse $V(t)$ and photon-echo intensity $I(t)$ during the time of the electric-pulse action. We determined the Stark shift from comparing the experimental and theoretical curves for each voltage pulse. Theoretical curves in Fig.1a uses (1) and Stark coefficient $\partial v / \partial E=0.1125 \mathrm{MHz} \cdot \mathrm{V}^{-1} \mathrm{~cm}$. This value is about $2 \%$ less then obtained in [5].

If the electric field pulse moves between the first and the second laser pulses or between the second pulse and an echo, a relative phase shift $\varphi$ does not depend on time and it is proportional to the

\footnotetext{
${ }^{a}$ Corresponding author: vlisin@kfti.knc.ru
} 
voltage pulse area. Echo intensity is oscillated, the echo waveform is not changed.
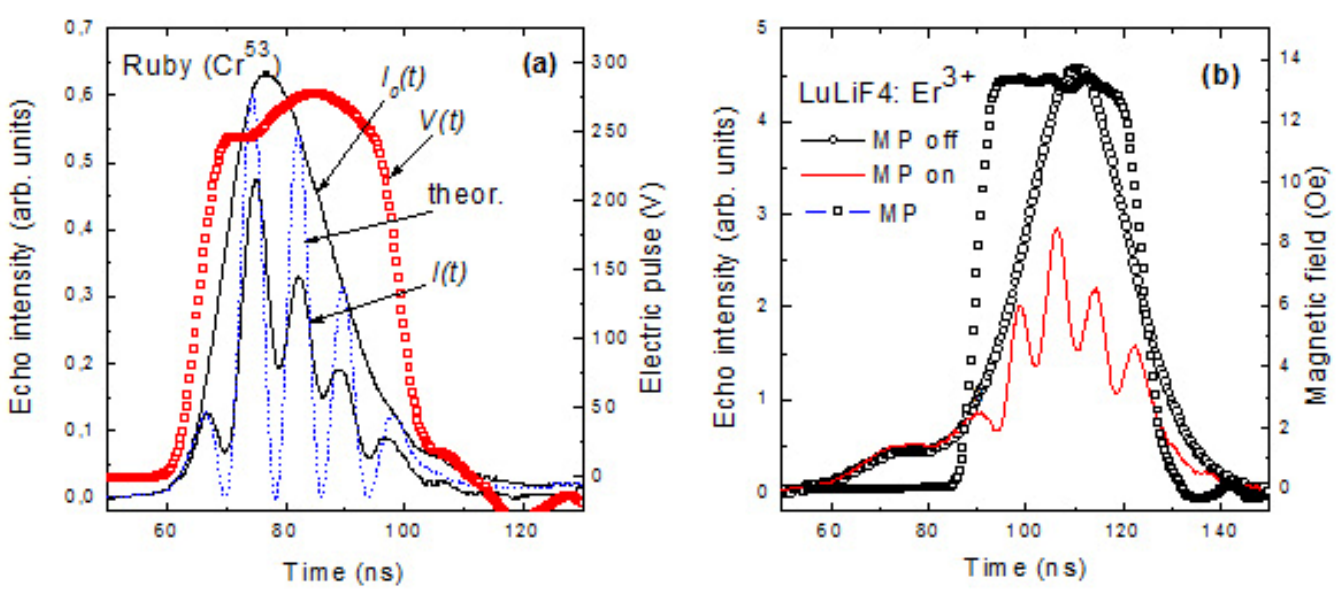

Figure 1. Modulation of the form of a photon echo under action: (a) - electric and (b) - a magnetic pulse in a ruby and $\mathrm{LuLiF}_{4}: \mathrm{Er}^{3+}$ accordingly.

In Fig. $1 \mathrm{~b}$ the similar curves are shown for the case of magnetic field pulse. Echo is excited by $\pi$ polarization light in transition ${ }^{4} \mathrm{I}_{15 / 2} \rightarrow{ }^{4} \mathrm{~F}_{9 / 2}$ of ion $\mathrm{Er}^{3+}$ in $\mathrm{LuLiF}_{4}$. The magnitudes of Zeeman coefficients determined in [2-4] are $\partial v_{\pi} / \partial E=4.578 \mathrm{MHz} / \mathrm{Oe}$ and 4.637 MHz/Oe in $\mathrm{LuLiF}_{4}$ and, accordingly, $\mathrm{YLiF}_{4}$. In [2-4] the according Zeeman coefficients were obtained $\partial v_{\sigma} / \partial E=8.858$ $\mathrm{MHz} / \mathrm{Oe}$ and $9.3045 \mathrm{MHz} / \mathrm{Oe}$ if photon echo is excited by a light of $\sigma$-polarizaion. Using this values, the g-factors of ground ${ }^{4} \mathrm{I}_{15 / 2}$ and excited ${ }^{4} \mathrm{~F}_{9 / 2}$ optical states of $\mathrm{Er}^{3+}$ ion in $\mathrm{LuLiF}_{4}$ and $\mathrm{YLiF}_{4}$ have been measured in [2-4]. The ground state g-factor values are in good agreement with those measured by EPR method: $\mathrm{g}\left(\mathrm{LuLiF}_{4}: \mathrm{Er}^{3+}\right)=3.09, \mathrm{~g}\left(\mathrm{YLiF}_{4}: \mathrm{Er}^{3+}\right)=3.14$.

The excited state $\mathrm{g}$-factor value $\mathrm{g}=9.76$ for $\mathrm{YLiF}_{4}: \mathrm{Er}^{3+}$ well coincides with the known values received by a method of optical absorption in strong magnetic fields. Value $\mathrm{g}\left({ }^{4} \mathrm{~F}_{9 / 2}\right)=9.6$ for $\mathrm{Er}^{3+}$ in $\mathrm{LuLiF}_{4}$ is obtained in [2-4] for the first time.

In our study we determined magnetic parameters of the paramagnetic ion with an accuracy comparable to the EPR and electric parameter of echo-actively ion using the known values of the magnetic and electric pulse amplitudes. If the magnetic and electric parameters are known, it is possible to determine the amplitude of the magnetic or electric pulse, using the relation (1). For example, if the magnetic or electric field is created by the pulse change of the dipole-dipole interaction of the ion with the environment. This can be used to determine the distance to the centers of the environment by measuring the waveform of the echo modulation periods, not only in the optical range, but also in the EPR and NMR bands.

This work was supported by the RAS program "Fundamental optical spectroscopy and its applications" and by the RFBR grants no. 14-02-00041a and 14-02-90000-Bel.a.

\section{References}

1. V. Lisin, A. Shegeda and K. Gerasimov, JETP Letters 95, 61-65 (2012)

2. V. Lisin, A. Shegeda, JETP Letters 96, 328-332 (2012)

3. V. Lisin, A. Shegeda and V. Samartsev, Laser Phys. Lett. 025701 (2015)

4. V. Lisin, A. Shegeda and V. Samartsev,, J. of Physics: Conf. Series 613, 012013 (2015)

5. M. G. Cohen and N. Bloembergen, Phys. Rev. 135, A950 (1964) 